\title{
Explaining Solar Neutrinos with Heavy Higgs Masses in Partial Split Supersymmetry
}

\author{
Marco Aurelio Díaz, Francisca Garay, and Benjamin Koch \\ Departamento de Física, Pontificia Universidad Católica de Chile, \\ Avenida Vicuña Mackenna 4860, Santiago, Chile
}

(Dated: November 2, 2018)

\begin{abstract}
Partial Split Supersymmetry with violation of R-parity as a model for neutrino masses is explored. It is shown that at the one-loop level the model can give predictions that are in agreement with all present experimental values for the neutrino sector. An analytical result is that the small solar neutrino mass difference can be naturally explained in the decoupling limit for the heavy Higgs mass eigenstates.
\end{abstract}

\section{INTRODUCTION}

Supersymmetric models softly broken at low energies introduce new sources of flavor changing neutral currents and new CP-violating phases that can create un-observed phenomenological effects. If supersymmetric particles are of the order of the electroweak scale, it is not obvious why they do not contribute to processes with flavor changing neutral currents with rates higher than observed [1]. Large values of sparticle masses is one mechanism that explains the inconspicuous contributions from supersymmetry to flavor observables. Split supersymmetric models were introduced advertising precisely this feature [2].

R-Parity violation in supersymmetric models [3] is an attractive feature because it provides a mechanism for neutrino mass generation. It is specially compelling in the case of Bilinear R-Parity violation (BRpV) [4] because an atmospheric mass difference is generated at tree-level by a low energy see-saw mechanism triggered by a mixing between neutrinos and neutralinos. In addition, a solar mass difference is generated at one-loop level by contributions from all particles [5], explaining the hierarchy between atmospheric and solar mass scales. Original Split Supersymmetry (SS) conserves R-Parity, nonetheless, the possibility has been explored, proving for example that a SS with R-Parity violation cannot generate a solar mass $[6,7,8,9]$.

In Partial Split Supersymmetry (PSS) [7], all sfermions are very heavy and decouple from the low energy theory, alleviating the flavor constraints present in supersymmetric models. Nevertheless, as opposed to the original Split Supersymmetric model, both Higgs boson doublets remain light in comparison with the split supersymmetric scale $\widetilde{m}$. Additionally we assume that R-Parity is not conserved. As a consequence, a solar neutrino mass difference is generated at one-loop, while the atmospheric mass difference is generated at 
tree level.

In contrast to gravitationally inspired models [10], in PSS the solar neutrino mass difference is generated by loops involving the CP-odd Higgs $A$ and the CP-even Higgs bosons $h$ and $H$. We are particularly interested in the limit $m_{A} \gg m_{Z}$, called the decoupling limit [11], where the light Higgs $h$ decouples from the heavy ones, acquiring SM-like couplings to fermions. Under this condition, the Higgs boson $h$ does not contribute to the solar mass, and it is experimentally challenging to distinguish it from the SM Higgs boson. In this article we study this scenario, and the ability of the mechanism to generate a solar mass from loops involving the heavy Higgs bosons $H$ and $A$.

\section{R-PARITY VIOLATION AND NEUTRINO MASSES IN PARTIAL SPLIT SUSY}

Partial Split Supersymmetry is an effective theory whose lagrangian is valid at scales lower than $\widetilde{m}$. The low energy lagrangian includes two Higgs doublet $H_{u}$ and $H_{d}$, and it is characterized by the following R-Parity conserving terms,

$$
\begin{aligned}
\mathcal{L}_{P S S}^{R p C} \ni & -\left[m_{1}^{2} H_{d}^{\dagger} H_{d}+m_{2}^{2} H_{u}^{\dagger} H_{u}-m_{12}^{2}\left(H_{d}^{T} \epsilon H_{u}+h . c .\right)\right. \\
& \left.+\frac{1}{2} \lambda_{1}\left(H_{d}^{\dagger} H_{d}\right)^{2}+\frac{1}{2} \lambda_{2}\left(H_{u}^{\dagger} H_{u}\right)^{2}+\lambda_{3}\left(H_{d}^{\dagger} H_{d}\right)\left(H_{u}^{\dagger} H_{u}\right)+\lambda_{4}\left|H_{d}^{T} \epsilon H_{u}\right|^{2}\right] \\
& +h_{u} \bar{u}_{R} H_{u}^{T} \epsilon q_{L}-h_{d} \bar{d}_{R} H_{d}^{T} \epsilon q_{L}-h_{e} \bar{e}_{R} H_{d}^{T} \epsilon l_{L}- \\
& -\frac{1}{\sqrt{2}} H_{u}^{\dagger}\left(\tilde{g}_{u} \sigma \widetilde{W}+\tilde{g}_{u}^{\prime} \widetilde{B}\right) \widetilde{H}_{u}-\frac{1}{\sqrt{2}} H_{d}^{\dagger}\left(\tilde{g}_{d} \sigma \widetilde{W}-\tilde{g}_{d}^{\prime} \widetilde{B}\right) \widetilde{H}_{d}+\text { h.c. }
\end{aligned}
$$

In the first two lines we have the Higgs potential, including both mass and self interaction terms. The electroweak symmetry is spontaneously broken as in the MSSM when the Higgs fields acquire non vanishing vacuum expectation values. In the third line we have the Yukawa interactions, and in the fourth line we have the interactions between Higgs, gauginos and higgsinos. This lagrangian is to be compared with the supersymmetric lagrangian valid above the scale $\widetilde{m}$, which includes the analogous terms,

$$
\begin{aligned}
\mathcal{L}_{\text {susy }}^{R p C} \ni & -\left[m_{1}^{2} H_{d}^{\dagger} H_{d}+m_{2}^{2} H_{u}^{\dagger} H_{u}-m_{12}^{2}\left(H_{d}^{T} \epsilon H_{u}+\text { h.c. }\right)+\frac{1}{8}\left(g^{2}+g^{\prime 2}\right)\left(H_{d}^{\dagger} H_{d}\right)^{2}\right. \\
& \left.+\frac{1}{8}\left(g^{2}+g^{\prime 2}\right)\left(H_{u}^{\dagger} H_{u}\right)^{2}+\frac{1}{4}\left(g^{2}-g^{\prime 2}\right)\left(H_{d}^{\dagger} H_{d}\right)\left(H_{u}^{\dagger} H_{u}\right)-\frac{1}{2} g^{2}\left|H_{d}^{T} \epsilon H_{u}\right|^{2}\right] \\
& +\lambda_{u} \bar{u}_{R} H_{u}^{T} \epsilon q_{L}-\lambda_{d} \bar{d}_{R} H_{d}^{T} \epsilon q_{L}-\lambda_{e} \bar{e}_{R} H_{d}^{T} \epsilon l_{L} \\
& -\frac{1}{\sqrt{2}} H_{u}^{\dagger}\left(g \sigma \widetilde{W}+g^{\prime} \widetilde{B}\right) \widetilde{H}_{u}-\frac{1}{\sqrt{2}} H_{d}^{\dagger}\left(g \sigma \widetilde{W}-g^{\prime} \widetilde{B}\right) \widetilde{H}_{d}+\text { h.c. }
\end{aligned}
$$

These two models are connected through boundary conditions at the scale $\widetilde{m}$. For the Higgs self couplings they are,

$$
\lambda_{1}=\lambda_{2}=\frac{1}{4}\left(g^{2}+g^{\prime 2}\right), \quad \lambda_{3}=\frac{1}{4}\left(g^{2}-g^{\prime 2}\right), \quad \lambda_{4}=-\frac{1}{2} g^{2},
$$


which are typical matching conditions between the MSSM and two Higgs doublet models. In an analogous way we have for the Yukawa couplings at $\widetilde{m}$,

$$
h_{u}=\lambda_{u}, \quad h_{d}=\lambda_{d}, \quad h_{e}=\lambda_{e},
$$

and for the higgsino-gaugino Yukawa couplings at $\widetilde{m}$,

$$
\tilde{g}_{u}=\tilde{g}_{d}=g, \quad \tilde{g}_{u}^{\prime}=\tilde{g}_{d}^{\prime}=g^{\prime}
$$

The two Higgs fields $H_{u}$ and $H_{d}$ acquire a vacuum expectation value $v_{u}$ and $v_{d}$, defining the usual mixing angle $\tan \beta=v_{u} / v_{d}$. The neutralino mass matrix in this scenario is written as,

$$
\mathbf{M}_{\chi^{0}}^{P S S}=\left[\begin{array}{cccc}
M_{1} & 0 & -\frac{1}{2} \tilde{g}_{d}^{\prime} v_{d} & \frac{1}{2} \tilde{g}_{u}^{\prime} v_{u} \\
0 & M_{2} & \frac{1}{2} \tilde{g}_{d} v_{d} & -\frac{1}{2} \tilde{g}_{u} v_{u} \\
-\frac{1}{2} \tilde{g}_{d}^{\prime} v_{d} & \frac{1}{2} \tilde{g}_{d} v_{d} & 0 & -\mu \\
\frac{1}{2} \tilde{g}_{u}^{\prime} v_{u} & -\frac{1}{2} \tilde{g}_{u} v_{u} & -\mu & 0
\end{array}\right] .
$$

In Partial Split Supersymmetry with non conserved R-Parity, neutrino masses are generated [7]. Trilinear $\mathrm{RpV}$ terms are irrelevant because high sfermions masses make their loop contributions negligible. Bilinear $\mathrm{RpV}$ terms do contribute via neutrino/neutralino mixing. The relevant terms are,

$$
\mathcal{L}_{P S S}^{R p V}=-\epsilon_{i} \widetilde{H}_{u}^{T} \epsilon L_{i}-\frac{1}{\sqrt{2}} b_{i} H_{u}^{T} \epsilon\left(\tilde{g}_{d} \sigma \widetilde{W}-\tilde{g}_{d}^{\prime} \widetilde{B}\right) L_{i}+\text { h.c. },
$$

where $\epsilon_{i}$ are the usual mass parameters that mix Higgs with lepton superfields, and $b_{i}$ are effective couplings between Higgs, gauginos and leptons. After the Higgs fields acquire vacuum expectation values, mixing terms are generated between neutrinos, on one hand, and higgsinos and gauginos on the other hand,

$$
\mathcal{L}_{P S S}^{R p V}=-\left[\epsilon_{i} \widetilde{H}_{u}^{0}+\frac{1}{2} b_{i} v_{u}\left(\tilde{g}_{d} \widetilde{W}_{3}-\tilde{g}_{d}^{\prime} \widetilde{B}\right)\right] \nu_{i}+\text { h.c. }+\ldots
$$

and they extend the $4 \times 4$ neutralino mass matrix, in eq. (6), to a $7 \times 7$ mass matrix that includes the neutrinos. The off-diagonal mixing block is,

$$
m^{P S S}=\left[\begin{array}{cccc}
-\frac{1}{2} \tilde{g}_{d}^{\prime} b_{1} v_{u} & \frac{1}{2} \tilde{g}_{d} b_{1} v_{u} & 0 & \epsilon_{1} \\
-\frac{1}{2} \tilde{g}_{d}^{\prime} b_{2} v_{u} & \frac{1}{2} \tilde{g}_{d} b_{2} v_{u} & 0 & \epsilon_{2} \\
-\frac{1}{2} \tilde{g}_{d}^{\prime} b_{3} v_{u} & \frac{1}{2} \tilde{g}_{d} b_{3} v_{u} & 0 & \epsilon_{3}
\end{array}\right]
$$

while the neutrino-neutrino block is a null $3 \times 3$ matrix. After a low energy see-saw mechanism, the effective neutrino mass matrix is,

$$
\mathbf{M}_{\nu}^{e f f}=-m^{P S S}\left(\mathrm{M}_{\chi^{0}}^{P S S}\right)^{-1}\left(m^{P S S}\right)^{T}=\frac{M_{1} \tilde{g}_{d}^{2}+M_{2}{\tilde{g^{\prime}}}_{d}^{2}}{4 \operatorname{det} M_{\chi^{0}}^{P S S}}\left[\begin{array}{ccc}
\Lambda_{1}^{2} & \Lambda_{1} \Lambda_{2} & \Lambda_{1} \Lambda_{3} \\
\Lambda_{2} \Lambda_{1} & \Lambda_{2}^{2} & \Lambda_{2} \Lambda_{3} \\
\Lambda_{3} \Lambda_{1} & \Lambda_{3} \Lambda_{2} & \Lambda_{3}^{2}
\end{array}\right],
$$


with $\Lambda_{i}=\mu b_{i} v_{u}+\epsilon_{i} v_{d}$, and with the determinant of the neutralino submatrix equal to,

$$
\operatorname{det} M_{\chi^{0}}^{P S S}=-\mu^{2} M_{1} M_{2}+\frac{1}{2} v_{u} v_{d} \mu\left(M_{1} \tilde{g}_{u} \tilde{g}_{d}+M_{2} \tilde{g}_{u}^{\prime} \tilde{g}_{d}^{\prime}\right)+\frac{1}{16} v_{u}^{2} v_{d}^{2}\left(\tilde{g}_{u}^{\prime} \tilde{g}_{d}-\tilde{g}_{u} \tilde{g}_{d}^{\prime}\right)^{2} .
$$

As it is well known, the neutrino mass matrix in eq. (10) has only one eigenvalue different from zero, and quantum corrections must be added in order to generate a solar mass.

\section{HIGGS DECOUPLING LIMIT}

The Higgs decoupling limit in the MSSM, or in the non-supersymmetric two Higgs doublet model 2HDM, is the regime where the lightest Higgs scalar mass is much smaller that all the other Higgs boson masses [11]. It has been studied in detail because if realized in nature, it will be experimentally difficult to distinguish the lithest Higgs boson properties from the ones of the SM Higgs boson.

If we neglect the running of the Higgs potential parameters, in first approximation the Higgs sector in PSS is analogous to the one in the MSSM. At tree level we have for the neutral CP-even Higgs bosons the following masses,

$$
m_{h, H}^{2}=\frac{1}{2}\left(m_{A}^{2}+m_{Z}^{2}\right) \mp \frac{1}{2} \sqrt{\left(m_{A}^{2}+m_{Z}^{2}\right)^{2}-4 m_{A}^{2} m_{Z}^{2} c_{2 \beta}^{2}}
$$

as a function of the neutral CP-odd Higgs mass $m_{A}$ and $\tan \beta$. Although $m_{h}$ receive large quantum corrections, they are negligible for the heavy Higgs $\mathrm{H}$ when $m_{A}$ is large. Thus the tree-level formula for $m_{H}$ is adequate in the decoupling limit. In this limit, when $m_{A} \gg m_{Z}$, we have

$$
m_{H}^{2} \approx m_{A}^{2}+m_{Z}^{2} \sin ^{2}(2 \beta)
$$

which is a very good approximation already for $m_{A}>200 \mathrm{GeV}$. The CP-even Higgs mass matrix is diagonalized by a rotation with an angle $\alpha$, which satisfies at tree-level,

$$
\cos ^{2}(\alpha-\beta)=\frac{m_{h}^{2}\left(m_{Z}^{2}-m_{h}^{2}\right)}{m_{A}^{2}\left(m_{H}^{2}-m_{h}^{2}\right)}
$$

The quantity $\cos (\alpha-\beta)$ is important because it is equal to the ratio between the heavy Higgs coupling to two $Z$ bosons (and two $W$ bosons) and the same couplings for the SM Higgs boson, $H_{S M}$. Thus, it is a key parameter for the determination of the $H$ production cross section in association with gauge bosons. Conversely, $\sin (\alpha-\beta)$ is proportional to the light Higgs boson couplings to two gauge bosons. In this limit we have,

$$
\cos ^{2}(\alpha-\beta) \approx \frac{m_{Z}^{4} \sin ^{2}(4 \beta)}{4 m_{A}^{4}}
$$


This means that $H$ decouples from the low energy theory. Thus the $h$ production cross section becomes ever more similar to the $H_{S M}$ one. The same happens to the $h$ couplings to fermions: they become similar to the $H_{S M}$ couplings in the decoupling limit, making it an experimental challenge to differentiate a $H_{S M}$ from a $h$ in this scenario.

\section{LOOP CORRECTIONS TO NEUTRINO MASSES IN THE DECOUPLING LIMIT}

In PSS the only loops capable to contribute to the solar mass are loops involving neutralinos and neutral Higgs bosons.

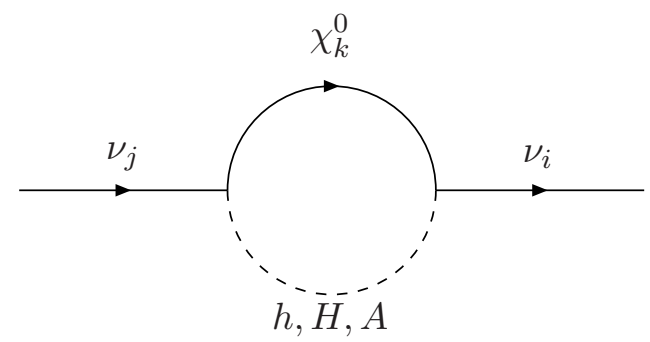

Radiative corrections to the effective neutrino mass matrix are of the form,

$$
\Delta \Pi_{i j}=A \Lambda_{i} \Lambda_{j}+B\left(\Lambda_{i} \epsilon_{j}+\Lambda_{j} \epsilon_{i}\right)+C \epsilon_{i} \epsilon_{j}
$$

where the $A$-term is the only one that receives tree-level contributions, as indicated in eq. (10). Charged and neutral gauge bosons contribute only to the $A$-term, i.e., to the atmospheric mass. The charged Higgs boson contributes to $A$ and $B$-terms. However, the $B$-term is scale dependent and can be rendered zero with an appropriate choice for the arbitrary substraction scale $Q$. Therefore, charged Higgs bosons do not contribute neither to the solar mass [7]. This leaves only the neutral Higgs bosons, whose contribution to the $C$ term are,

$$
\begin{aligned}
\left.\Delta \Pi_{i j}^{h, H}\right|_{\epsilon \epsilon} & =-\frac{1}{16 \pi^{2}} \sum_{k=1}^{4}\left(F_{k}^{h, H}\right)^{2} \epsilon_{i} \epsilon_{j} m_{\chi_{k}^{0}} B_{0}\left(0 ; m_{\chi_{k}^{0}}^{2}, m_{h, H}^{2}\right), \\
\left.\Delta \Pi_{i j}^{A}\right|_{\epsilon \epsilon} & =\frac{1}{16 \pi^{2}} \sum_{k=1}^{4}\left(F_{k}^{A}\right)^{2} \epsilon_{i} \epsilon_{j} m_{\chi_{k}^{0}} B_{0}\left(0 ; m_{\chi_{k}^{0}}^{2}, m_{A}^{2}\right)
\end{aligned}
$$

where the sum is over all four neutralinos, with masses $m_{\chi_{k}^{0}}$, and $B_{0}$ is the usual Veltman function for twopoint Green functions. Note that $h$ and $H$ contributions have an overall minus sign, while the $A$ contribution 
has not. The reason is that $A$ has CP-odd couplings to fermions. The couplings $F_{k}$ are equal to,

$$
\begin{aligned}
F_{k}^{h} & =\frac{\cos (\alpha-\beta)}{2 \mu s_{\beta}}\left(g N_{k 2}^{*}-g^{\prime} N_{k 1}^{*}\right), \\
F_{k}^{H} & =\frac{\sin (\alpha-\beta)}{2 \mu s_{\beta}}\left(g N_{k 2}^{*}-g^{\prime} N_{k 1}^{*}\right), \\
F_{k}^{A} & =\frac{1}{2 \mu s_{\beta}}\left(g N_{k 2}^{*}-g^{\prime} N_{k 1}^{*}\right) .
\end{aligned}
$$

The factor in the parenthesis indicates that it is the gaugino component of the neutralinos the one that contributes to the solar neutrino mass. In addition, the $B_{0}$ Veltman's function for zero external momentum is,

$$
B_{0}\left(0 ; M^{2}, m^{2}\right)=\Delta+1-\frac{M^{2} \ln \frac{M^{2}}{Q^{2}}-m^{2} \ln \frac{m^{2}}{Q^{2}}}{M^{2}-m^{2}}
$$

where $Q$ is the arbitrary substraction scale and $\Delta$ is the regulator. In this way, the one-loop contribution to the $C$ coefficient of the $\epsilon_{i} \epsilon_{j}$ term in eq. 16 is,

$$
\begin{aligned}
C^{A H h}= & \frac{1}{64 \pi^{2} \mu^{2} s_{\beta}^{2}} \sum_{k=1}^{4} m_{\chi_{k}^{0}}\left(g N_{k 2}-g^{\prime} N_{k 1}\right)^{2} \\
& {\left[B_{0}\left(0 ; m_{\chi_{k}^{0}}^{2}, m_{A}^{2}\right)-\sin ^{2}(\alpha-\beta) B_{0}\left(0 ; m_{\chi_{k}^{0}}^{2}, m_{H}^{2}\right)-\cos ^{2}(\alpha-\beta) B_{0}\left(0 ; m_{\chi_{k}^{0}}^{2}, m_{h}^{2}\right)\right] . }
\end{aligned}
$$

The fact that the divergent term in $B_{0}$ is independent of all masses implies that the $C$ coefficient is finite, which in turn leads to the finiteness of the solar mass.

The neutrino masses generated from eq. (16) include a massless neutrino $m_{\nu_{1}}=0$, and the two massive ones given by,

$$
m_{\nu_{3,2}}=\frac{1}{2}\left(A|\vec{\Lambda}|^{2}+C|\vec{\epsilon}|^{2}\right) \pm \frac{1}{2} \sqrt{\left(A|\vec{\Lambda}|^{2}+C|\vec{\epsilon}|^{2}\right)^{2}-4 A C|\vec{\Lambda} \times \vec{\epsilon}|^{2}}
$$

where the sign is chosen such that $\left|m_{\nu_{2}}\right|<\left|m_{\nu_{3}}\right|$. We are interested in the behavior of the solar mass in the Higgs decoupling limit, where $m_{A} \gg m_{Z}$. The $B_{0}$ Veltman function in eq. (19) has the following expansion when one of the masses is much larger than the other, $M \gg m$,

$$
B_{0}\left(0 ; M^{2}, m^{2}\right) \approx \Delta-\ln \frac{M^{2}}{Q^{2}}+1-\frac{m^{2}}{M^{2}} \ln \frac{M^{2}}{m^{2}}-\frac{m^{4}}{M^{4}} \ln \frac{M^{2}}{m^{2}}
$$

In the regime where the neutralinos are much lighter than the CP-odd Higgs mass, we find for the $C$ coefficient,

$$
C^{A H h} \approx \frac{m_{Z}^{2} \sin ^{2} 2 \beta}{64 \pi^{2} \mu^{2} s_{\beta}^{2} m_{A}^{2}} \sum_{k=1}^{4} m_{\chi_{k}^{0}}\left(g N_{k 2}-g^{\prime} N_{k 1}\right)^{2}
$$


where the term in parenthesis correspond to the zino component of each neutralino. This motivates us to define,

$$
\left\langle m_{\widetilde{Z}}\right\rangle \equiv \sum_{k=1}^{4} m_{\chi_{k}^{0}}\left(c_{W} N_{k 2}-s_{W} N_{k 1}\right)^{2}
$$

as the zino effective mass. In this way, we find that the CP-odd Higgs mass is related to the solar neutrino mass difference by the following simple relation,

$$
m_{A}^{2} \approx \frac{g^{4} m_{Z}^{2} \cos ^{2} \beta}{64 \pi^{2} c_{W}^{4}} \frac{\left\langle m_{\widetilde{Z}}\right\rangle m_{\widetilde{\gamma}}}{M_{1} M_{2}} \sqrt{\frac{\delta}{1+\delta}} \frac{|\vec{\Lambda} \times \vec{\epsilon}|^{2}}{\mu^{4} \Delta m_{\text {sol }}^{2}}
$$

where $\delta=\Delta m_{\text {sol }}^{2} / \Delta m_{\text {atm }}^{2} \approx 0.035$ and $m_{\widetilde{\gamma}}=c_{W}^{2} M_{1}+s_{W}^{2} M_{2}$ is the photino mass.

This formula is remarkable. First we notice that the CP-odd Higgs mass squared is inversely proportional to the solar mass difference. The reason behind this feature is as follows. In the Higgs decoupling limit the light Higgs has SM-like couplings and does not contribute to the solar mass. More precisely, $h$ contribution to the neutrino mass matrix is proportional to $\cos ^{2}(\alpha-\beta)$, which rapidly approaches zero as $m_{A} \gg m_{Z}$, as indicated by eq. (15). The other two neutral Higgs bosons, $H$ and $A$, have large contributions to the neutrino mass matrix, but with opposite signs, and as it can be seen already from eq. (17) they tend to cancel each other in the decoupling limit. Therefore, the fact that Supersymmetry forces $m_{H} \rightarrow m_{A}$ when $m_{A} \gg m_{Z}$, produces a fine cancellation that eventually generates a small solar mass. This fine cancellation is not a fine-tuning because it is a cancellation forced by symmetry. In addition, the CP-odd Higgs mass is dependent on the atmospheric mass through the ratio $\delta$ between solar at atmospheric scales. Thus, the atmospheric mass also affects $m_{A}^{2}$ inversely although in an indirect way. Our model explains the smallness of $\delta$ because the atmospheric mass is generated at tree-level while the solar mass is generated at one-loop.

The CP-odd squared mass is proportional to $|\vec{\Lambda} \times \vec{\epsilon}|$, with an extra term $\mu^{4}$ in the denominator. This cross product is there because if $\vec{\epsilon}$ and $\vec{\Lambda}$ are parallel, the symmetry of the neutrino mass matrix observed at tree-level in eq. (11) is not removed, and no solar mass is generated. Furthermore, $m_{A}^{2}$ is proportional to the $Z$ boson mass, indicating that a Majorana neutrino mass needs not only R-Parity violation but a broken $S U(2)_{L}$ symmetry as well. Additionally, $m_{A}^{2}$ is proportional to $\cos ^{2} \beta$ because the relevant vacuum expectation value is $\left\langle H_{d}\right\rangle$.

We also notice that the CP-odd Higgs mass squared is proportional to the effective zino mass and to the photino mass, normalized by both relevant gaugino masses. The appearance of the photino mass is due to the tree-level contribution to the effective neutrino mass matrix. The origin of the zino mass is that it is the zino component of each neutralino the one that contributes to the neutrino mass matrix, and it multiplies the neutralino masses from the fermionic propagator. In addition, for this mechanism to work, R-Parity must be broken, and the neutrino mixing with down higgsinos in the one hand, and Higgs bosons mixing with 
sneutrinos in the other hand, are crucial. The following schematic diagram, corresponding to the heavy Higgs boson loops, may help to understand the origin of the one-loop contributions to the neutrino mass matrix,

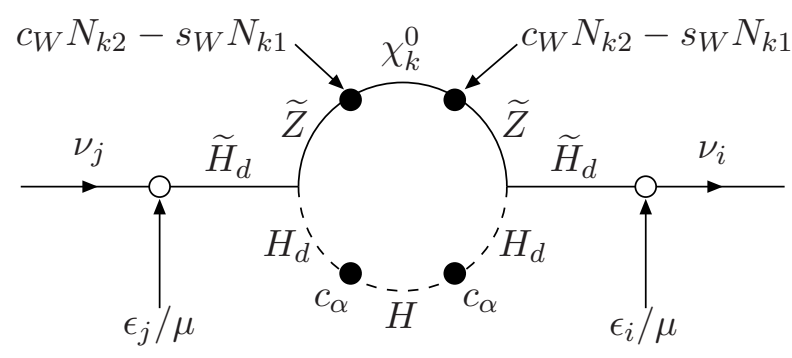

The supersymmetric vertex behind this diagram is the zino coupling to down-Higgs and down-higgsino. For this reason, the zino component from each neutralino is selected, $c_{W} N_{k 2}-s_{W} N_{k 1}$, which weights the corresponding neutralino mass picked up from the propagator. In addition, the down-Higgs component from the heavy Higgs $H$, given by $c_{\alpha}$, is selected. These mixings are represented in the diagram by full circles, as oppose to open circles which violate R-Parity. Indeed, R-Parity is violated at the mixing between neutrinos and down-higgsinos. These two mixings at the external legs also violate lepton number by two units, as it should be for a Majorana neutrino mass.

The second diagram is,

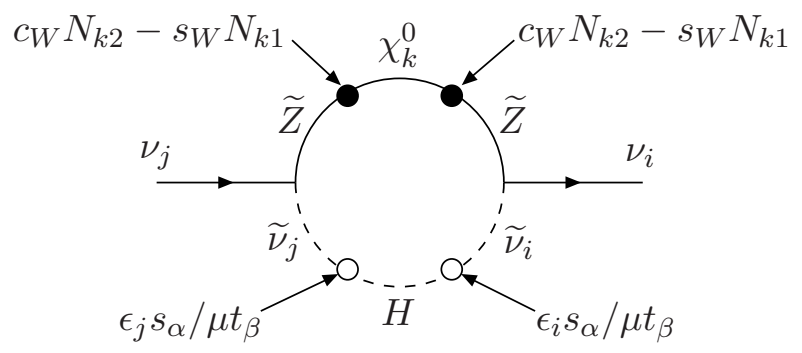

where the supersymmetric vertex supporting the diagram is the zino coupling to a neutrino and a sneutrino. The presence of the zino component of each neutralino is explained by the same argument as before. But in this case, R-Parity and lepton number violation appear in the Higgs boson mixing with sneutrinos. The magnitude of this mixing, indicated in the diagram, is explained in ref. [7]. Diagrams with one of each supersymmetric vertices also contribute, but are not shown. 
TABLE I: PSS and RpV parameters and neutrino observables for the working scenario $\mathrm{P}_{t}$

\begin{tabular}{|c|c|c|c|}
\hline Susy-Parameter & $\mathrm{P}_{t}$ & Scanned range & Units \\
\hline $\tan \beta$ & 10 & {$[2,50]$} & - \\
\hline$|\mu|$ & 450 & {$[0,1000]$} & $\mathrm{GeV}$ \\
\hline$M_{2}$ & 300 & {$[80,1000]$} & $\mathrm{GeV}$ \\
\hline$M_{1}$ & 150 & $M_{2} / 2$ & $\mathrm{GeV}$ \\
\hline$m_{h}$ & 120 & {$[114,140]$} & $\mathrm{GeV}$ \\
\hline$m_{A}$ & 1000 & {$[50,6000]$} & $\mathrm{GeV}$ \\
\hline$Q$ & 951.7 & - & $\mathrm{GeV}$ \\
\hline \multicolumn{4}{|l|}{ RpV-Parameter } \\
\hline$\epsilon_{1}$ & 0.0346 & - & $\mathrm{GeV}$ \\
\hline$\epsilon_{2}$ & 0.2516 & {$[-1,1]$} & $\mathrm{GeV}$ \\
\hline$\epsilon_{3}$ & 0.3504 & {$[-1,1]$} & $\mathrm{GeV}$ \\
\hline$\Lambda_{1}$ & -0.0259 & {$[-1,1]$} & $\mathrm{GeV}^{2}$ \\
\hline$\Lambda_{2}$ & -0.0011 & - & $\mathrm{GeV}^{2}$ \\
\hline$\Lambda_{3}$ & 0.0709 & {$[-1,1]$} & $\mathrm{GeV}^{2}$ \\
\hline
\end{tabular}

\begin{tabular}{ccc}
\hline Observable & Solution & Units \\
\hline \hline$\Delta m_{\text {atm }}^{2}$ & $2.45 \times 10^{-3}$ & $\mathrm{eV}^{2}$ \\
$\Delta m_{\text {sol }}^{2}$ & $7.9 \times 10^{-5}$ & $\mathrm{eV}^{2}$ \\
$\tan ^{2} \theta_{\text {atm }}$ & 0.824 & - \\
$\tan ^{2} \theta_{\text {sol }}$ & 0.487 & - \\
$\tan ^{2} \theta_{13}$ & 0.027 & - \\
$m_{e e}$ & 0.0016 & $\mathrm{eV}$ \\
\hline \hline
\end{tabular}

\section{NUMERICAL RESULTS}

In our numerical analysis the contributions to the neutrino mass matrix in eq. (16) were calculated and their influence on the neutrino observables such as mass differences and mixing angles was studied. The agreement with the experimental boundaries $(3 \sigma)[12]$ was quantified by calculating

$$
\chi^{2}=\left(\frac{10^{3} \Delta m_{a t m}^{2}-2.4}{0.4}\right)^{2}+\left(\frac{10^{5} \Delta m_{\text {sol }}^{2}-7.7}{0.6}\right)^{2}+\left(\frac{\sin ^{2} \theta_{a t m}-0.505}{0.165}\right)^{2}+\left(\frac{\sin ^{2} \theta_{\text {sol }}-0.33}{0.07}\right)^{2} .
$$

Additionally it was demanded that the upper bounds $\sin ^{2} \theta_{\text {reac }}<0.05$ and $m_{\beta \beta}<0.84 \mathrm{eV}$ have to be fulfilled. Thus, the model is in agreement with the experimental values if $\chi^{2}<4$. As a working scenario, we select a typical point in the PSS parameter space, denoted $\mathrm{P}_{t}$. This scenario consists of fixed values for the gaugino and higgsino mass parameters, $\tan \beta$, the light $\mathrm{CP}$-even and $\mathrm{CP}$-odd Higgs masses, and the BRpV parameters, all given in table I. This scenario satisfies the neutrino experimental constraints, predicting atmospheric and solar mass differences and mixing angles well within the $3 \sigma$ regions indicated in eq. (26). In addition, the predicted reactor angle and the neutrinoless double-beta decay mass parameter are below the upper bound. All these prediction of our $\mathrm{P}_{t}$ scenario are given in table $\square$

Next we scan the parameter space varying the PSS parameters according to the intervals indicated in 
table I] In Fig. 1] we vary $\epsilon_{2}$ and $\epsilon_{3}$ keeping all the other parameters as in $\mathrm{P}_{t}$, and plot the logarithm

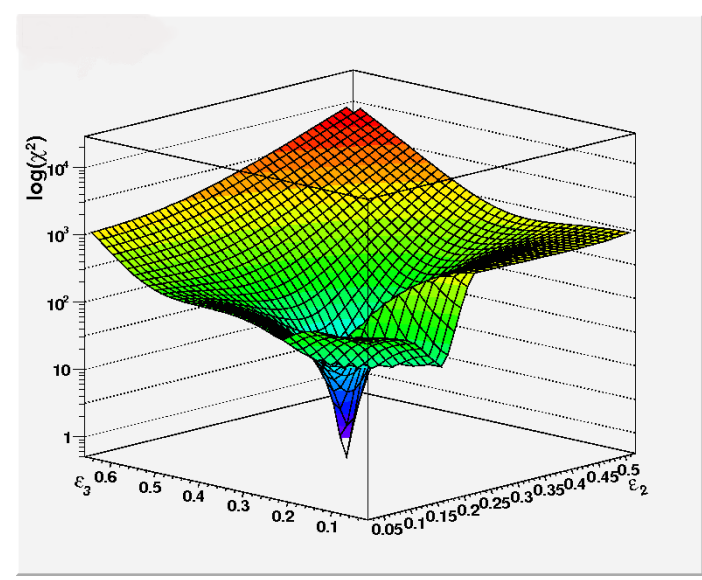

FIG. 1: $\chi^{2}$ in dependence of $\epsilon_{2}$ and $\epsilon_{3}$, while the other parameters are fixed around the central value from table प

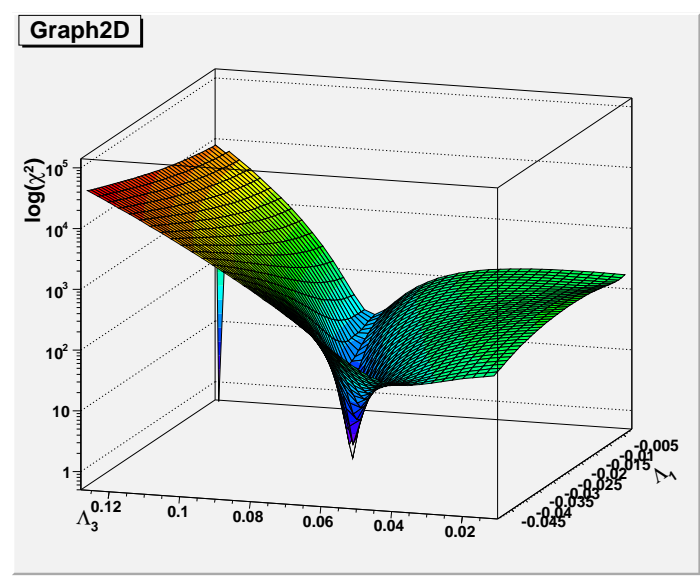

FIG. 2: $\chi^{2}$ in dependence of $\Lambda_{1}$ and $\Lambda_{3}$, while the other parameters are fixed around the central value from table $\llbracket$

of $\chi^{2}$ associated to each point in parameter space. Solutions with $\chi^{2}<4$ are clearly visible, they are compatible with experiments, and $\mathrm{P}_{t}$ is inside this region. The value for $\chi^{2}$ grows fast as we deviate from the experimentally accepted region around $\mathrm{P}_{t}$. In Fig. 2 we see the dependence on $\Lambda_{1}$ and $\Lambda_{3}$ while keeping the rest of the PSS parameters as indicated by $\mathrm{P}_{t}$. Again there is a steep growth on $\chi^{2}$ when we deviate from the experimentally accepted region. Of course, these experimentally compatible regions move around in the $\epsilon_{2}-\epsilon_{3}$ and $\Lambda_{1}-\Lambda_{3}$ planes when we change the fixed values of the other PSS parameters.

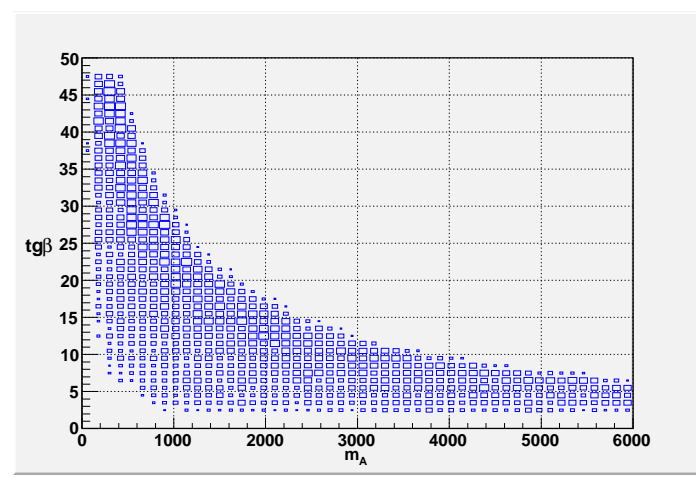

FIG. 3: Relation between $\tan \beta$ and $m_{A}$, while varying the other parameters as indicated in $\square$

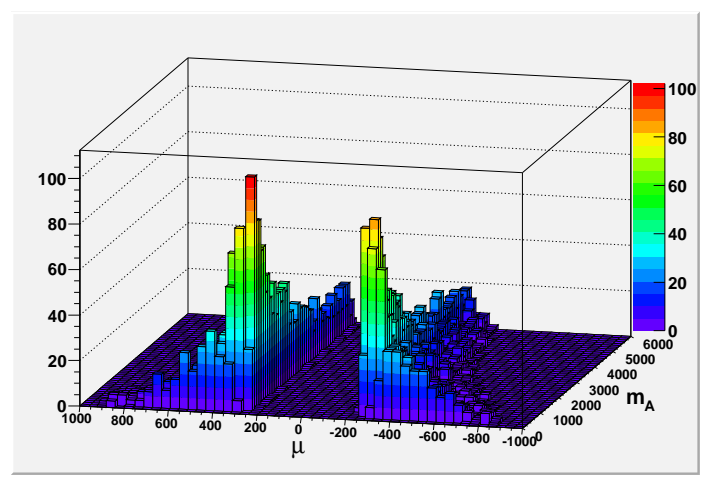

FIG. 4: Frequency plot in the $\mu-m_{A}$ plane, for points in parameter space that are consistent with neutrino experiments.

For the scan shown in Figs. 3 and 4 we keep the BRpV parameters $\epsilon_{i}$ and $\Lambda_{i}$ fixed to their $\mathrm{P}_{t}$ values, and vary the rest of the PSS parameters as indicated in table I] In Fig. 3 we have the relation between 


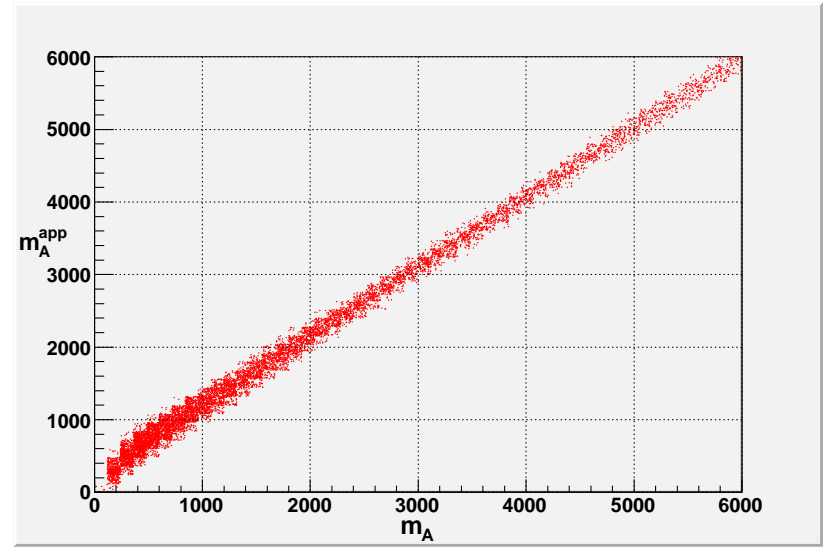

FIG. 5: Approximated $m_{A}^{\text {app }}$ as a function of its exact value $m_{A}$, for a scan with $10^{4}$ random points.

$\tan \beta$ and the $\mathrm{CP}$-odd Higgs mass $m_{A}$. The scan shows that an agreement with the neutrino observables is disfavored if both susy parameters $\tan \beta$ and $m_{A}$ take simultaneously large values. Due to this correlation a measurement of the value of one of the two parameters might give important information on the value of the other, even if the rest of the susy parameters are not known. A detailed study shows that the excluded region in the $\left(\tan \beta-m_{A}\right)$ plane comes due to the observed constraint on $\tan ^{2} \theta_{a t m}$. In Fig. 4 we show the relation between the higgsino mass parameter $\mu$ and $m_{A}$ from the same scan as before. This reveals that for large $m_{A} \approx 6000 \mathrm{GeV}$ good results compatible with neutrino experiments are only obtained for $200<|\mu|<350 \mathrm{GeV}$, whereas for smaller $m_{A} \approx 1000 \mathrm{GeV}$ the preferred region for $\mu$ widens up to $200<|\mu|<600 \mathrm{GeV}$. A detailed study shows that the observed values for $\Delta m_{\text {sol }}^{2}$ and $\Delta m_{\text {atm }}^{2}$ forbid solutions with $|\mu|<200 \mathrm{GeV}$. Finally, in order to test the accuracy of the approximated formula given in eq. (25), we perform a scan with $10^{4}$ random points. In Fig. 5 we include the points that satisfy the neutrino experimental constraints, and we see that the approximated formula works in a very large range of $m_{A}$ values, with larger percentage errors for smaller $m_{A}$, which is expected.

\section{SUMMARY}

This paper explored Partial Split Supersymmetry with $\mathrm{RpV}$ as a model for neutrino masses. It was shown that at the one-loop level the model can give predictions that are in very good agreement with all present experimental values for the neutrino sector. In contrast to this good agreement in PSS, it is not possible to generate all the neutrino mass parameters correctly in standard Split Supersymmetry with RpV. The difference between both models, lies solely in the fact that PSS allows for a larger Higgs sector, which contains the mass eigenstates $A$ and $H$ in addition the standard model like state $h$. A continuous transition from PSS to SS can be achieved by raising the values for the heavy Higgs masses $m_{A}$ and $m_{H}$ (Higgs 
decoupling limit). An analytical study of this limit $\left(m_{A} \gg m_{h}\right)$ reveals an approximate formula for PSS in which a large value for $m_{A}$ is directly connected to a small solar neutrino mass difference $\Delta m_{s o l}^{2}$. Therefore, the small observed value for $\Delta m_{\text {sol }}^{2}$ favors large values of $m_{A}$ up to $6 \mathrm{TeV}$. Such large values for $m_{A}$ would make PSS virtually indistinguishable from SS by using any observable other than neutrino masses.

Note Added: While this article was being written, we read the paper "SUSY Splits, But Then Returns" (arXiv:0909.5430) by Prof. Raman Sundrum [13], where models with two light Higgs doubles are also referred to as Partial Split Supersymmetry.

\section{Acknowledgments}

The work of M.A.D. was partly funded by Conicyt-PBCT grant No. ACT028 (Anillo Centro de Estudios Subatómicos), and by Conicyt-PBCT grant ACI35. B.K. was funded by Conicyt-PBCT grant PSD73.

[1] Y. Nir, arXiv:0708.1872 [hep-ph]; J. L. Feng, C. G. Lester, Y. Nir and Y. Shadmi, Phys. Rev. D 77, 076002 (2008) [arXiv:0712.0674[hep-ph]].

[2] N. Arkani-Hamed and S. Dimopoulos, JHEP 0506, 073 (2005) [arXiv:hep-th/0405159]; G. F. Giudice and A. Romanino, Nucl. Phys. B 699, 65 (2004) [Erratum-ibid. B 706, 65 (2005)] [arXiv:hep-ph/0406088].

[3] R. Barbier et al., Phys. Rept. 420, 1 (2005) |arXiv:hep-ph/0406039]; H. K. Dreiner, arXiv:hep-ph/9707435.

[4] R. Hempfling, Nucl. Phys. B 478, 3 (1996) [arXiv:hep-ph/9511288]; M. A. Diaz, M. Hirsch, W. Porod, J. C. Romao and J. W. F. Valle, Phys. Rev. D 68, 013009 (2003) [Erratum-ibid. D 71, 059904 (2005)] [arXiv:hep-ph/0302021]; M. Drees, S. Pakvasa, X. Tata and T. ter Veldhuis, Phys. Rev. D 57, 5335 (1998) arXiv:hep-ph/9712392]; F. Borzumati, Y. Grossman, E. Nardi and Y. Nir, Phys. Lett. B 384, 123 (1996) arXiv:hep-ph/9606251]; E. J. Chun, S. K. Kang, C. W. Kim and U. W. Lee, Nucl. Phys. B 544, 89 (1999) arXiv:hep-ph/9807327]; Y. Grossman and S. Rakshit, Phys. Rev. D 69, 093002 (2004) arXiv:hep-ph/0311310; S. Davidson and M. Losada, JHEP 0005, 021 (2000) [arXiv:hep-ph/0005080]; A. Dedes, S. Rimmer and J. Rosiek, JHEP 0608, 005 (2006) [arXiv:hep-ph/0603225].

[5] M. Hirsch, M. A. Diaz, W. Porod, J. C. Romao and J. W. F. Valle, Phys. Rev. D 62, 113008 (2000) [Erratum-ibid. D 65, 119901 (2002)] [arXiv:hep-ph/0004115].

[6] E. J. Chun and S. C. Park, "Neutrino mass from R-parity violation in split supersymmetry,” JHEP 0501 (2005) 009 [arXiv:hep-ph/0410242].

[7] M. A. Diaz, P. Fileviez Perez and C. Mora, Phys. Rev. D 79, 013005 (2009) [arXiv:hep-ph/0605285].

[8] S. Davidson and M. Losada, "Basis independent neutrino masses in the R(p) violating MSSM," Phys. Rev. D 65, 075025 (2002) arXiv:hep-ph/0010325.

[9] S. Davidson and M. Losada, "Neutrino masses in the R(p) violating MSSM," JHEP 0005, 021 (2000) 
[arXiv:hep-ph/0005080]; Y. Grossman and S. Rakshit, "Neutrino masses in R-parity violating supersymmetric models," Phys. Rev. D 69, 093002 (2004) [arXiv:hep-ph/0311310].

[10] E. K. Akhmedov, Z. G. Berezhiani, G. Senjanovic and Z. j. Tao, Phys. Rev. D 47, 3245 (1993) arXiv:hep-ph/9208230]; E. K. Akhmedov, Z. G. Berezhiani and G. Senjanovic, Phys. Rev. Lett. 69, 3013 (1992) arXiv:hep-ph/9205230;; A. de Gouvea and J. W. F. Valle, Phys. Lett. B 501, 115 (2001) arXiv:hep-ph/0010299;; V. Berezinsky, M. Narayan and F. Vissani, JHEP 0504, 009 (2005) arXiv:hep-ph/0401029]; A. Dighe, S. Goswami and W. Rodejohann, Phys. Rev. D 75, 073023 (2007) arXiv:hep-ph/0612328]; M. A. Diaz, B. Koch and B. Panes, Phys. Rev. D 79, 113009 (2009) [arXiv:0902.1720 [hep-ph]].

[11] J. F. Gunion and H. E. Haber, Phys. Rev. D 67, 075019 (2003) arXiv:hep-ph/0207010; H. E. Haber, M. J. Herrero, H. E. Logan, S. Penaranda, S. Rigolin and D. Temes, Phys. Rev. D 63, 055004 (2001) arXiv:hep-ph/0007006; A. Dobado, M. J. Herrero and S. Penaranda, Eur. Phys. J. C 17, 487 (2000) [arXiv:hep-ph/0002134].

[12] M. Maltoni, T. Schwetz, M. A. Tortola and J. W. F. Valle, "Status of global fits to neutrino oscillations," New J. Phys. 6 (2004) 122. [arXiv:hep-ph/0405172].

[13] R. Sundrum, arXiv:0909.5430 [hep-th]. 